\title{
Near-field hyperspectral quantum probing of multimodal plasmonic resonators
}

\author{
A. Cuche,${ }^{1, *}$ M. Berthel, ${ }^{2}$ U. Kumar, ${ }^{1}$ G. Colas des Francs,${ }^{3}$ S. Huant, ${ }^{2}$ E. Dujardin, ${ }^{1}$ C. Girard,${ }^{1}$ and A. Drezet ${ }^{2, *}$ \\ ${ }^{1}$ CEMES, University of Toulouse and CNRS (UPR 8011), 29 rue Jeanne Marvig, BP 94347, 31055 Toulouse, France \\ ${ }^{2}$ Université Grenoble Alpes, CNRS, Institut Néel, F-38000 Grenoble, France \\ ${ }^{3}$ Laboratoire Interdisciplinaire Carnot de Bourgogne (ICB) UMR 6303, \\ CNRS-Université Bourgogne Franche-Comté 9 Av. A. Savary, BP 47 870, France
}

(Received 28 June 2016; revised manuscript received 22 November 2016; published 10 March 2017)

\begin{abstract}
Quantum systems, excited by an external source of photons, display a photodynamics that is ruled by a subtle balance between radiative or nonradiative energy channels when interacting with metallic nanostructures. We apply and generalize this concept to achieve a quantum probing of multimodal plasmonic resonators by collecting and filtering the broad emission spectra generated by a nanodiamond (ND) hosting a small set of nitrogen-vacancy (NV) color centers attached at the apex of an optical tip. Spatially and spectrally resolved information on the photonic local density of states (ph-LDOS) can be recorded with this technique in the immediate vicinity of plasmonic resonators, paving the way for a complete near-field optical characterization of any kind of nanoresonators in the single photon regime.
\end{abstract}

DOI: 10.1103/PhysRevB.95.121402

The optical concepts developed in the research field of plasmonics have triggered practical applications for the miniaturization and the integration of optical devices as well as for molecular sensing applications. Particularly, these new devices offer interesting opportunities for optical exchange information with or between quantum systems [1].

Recently, systems consisting of isolated molecules in interaction with noble metal nanostructures have been used as prototype models to test and validate new proposal of active plasmonic devices [2-6]. These experiments use the excitation of surface plasmons to enhance and control the efficiency of the light transfer between molecular sites and remote detectors. In this context, a new breakthrough has been realized where nonclassical photon correlations from the emission of a quantum emitter coupled to a plasmonic structure have been demonstrated [7-10]. Aiming at improving the coupling efficiency and the reversibility of the emitters positioning, an elegant approach has been developed where single fluorescent particles are grafted at the apex of optical near-field tips. Historically, the use of a single emitter to achieve near-field optical mapping began with the pioneering work by Sandoghdar et al. where a near-field scanning optical microscope (NSOM) was designed with a source of light reduced to a single fluorescent molecule embedded in a microcrystal glued at the apex of the tip [11]. This seminal experiment triggered a strong interest for such near-field active probes that led to the spatially and time-resolved imaging of nanostructures $[12,13]$, or to the mapping of the magnetic local density of states [14], with systems based on molecules or rare-earth ions.

Nitrogen-vacancy colored centers in NDs have been particularly used as active probes because of the appealing optical properties in the visible of these photostable quantum emitters. Attached at the apex of a tip, they have been used to perform near-field optical mapping of metal nanostructures $[15,16]$, magnetic field imaging [17], on-demand single plasmon generation [18], and second-order quantum coherence

*aurelien.cuche@cemes.fr, aurelien.drezet@grenoble.cnrs.fr measurements of surface plasmon polaritons $[19,20]$. Since the size of such ultimate probe can be viewed as pointlike when compared to the variations of the optical fields, the physical interpretation of the mechanism causing the optical imaging process highlights the role of the ph-LDOS at the emitter position [21]. Time-resolved measurements have recently revealed the ph-LDOS around simple metallic nanostructures $[22,23]$. However, the vectorial nature of the single NV centers used in these experiments only probed a partial ph-LDOS projected along the dipole direction of the emitter. In addition, these projected ph-LDOS are averaged over the broad spectral range of the NV emission.

In this Rapid Communication, we develop a novel modal imaging concept, named Hyperspectral Quantum Near-Field Scanning Optical Microscope (HQ-NSOM), by demonstrating how the use of photochemically stable NV-based probes coupled to plasmonic cavities can lead to a genuine hyperspectral imaging of plasmonic modal topologies. As depicted in Fig. 1, an optically active probe, comprising a small number of $p$ independent $\mathrm{NV}^{-}$quantum emitters attached to an optical fiber, acts as a scalar single photon source with stable spectral emission wavelength ranging from 640 to $800 \mathrm{~nm}$ [see spectrum in Fig. 1(b)]. In particular, we show that a judicious filtering of this broad emission spectra enables to record images revealing the photonic local density of states (ph-LDOS) for different selected wavelengths by scanning the probe tip in the immediate vicinity of plasmonic resonators. The active NSOM probe proposed here contains a few NV centers so that it gives access to numerous parameters: namely, spectral imaging, radiative contribution to the photonic LDOS and indirectly to the full photonic LDOS. Moreover, it appears as a scalar probe so that we image the full mode profile. Finally, taking advantage of the few emitters, the active probe is a quantum optical source, opening the door to the characterization and design of quantum nanophotonics components, beyond plasmonics.

To highlight the photophysical mechanisms relevant for frequency-dependent ph-LDOS maps detection, we first introduce some fundamental equations associated with light emission from a quantum system. The total fluorescence 

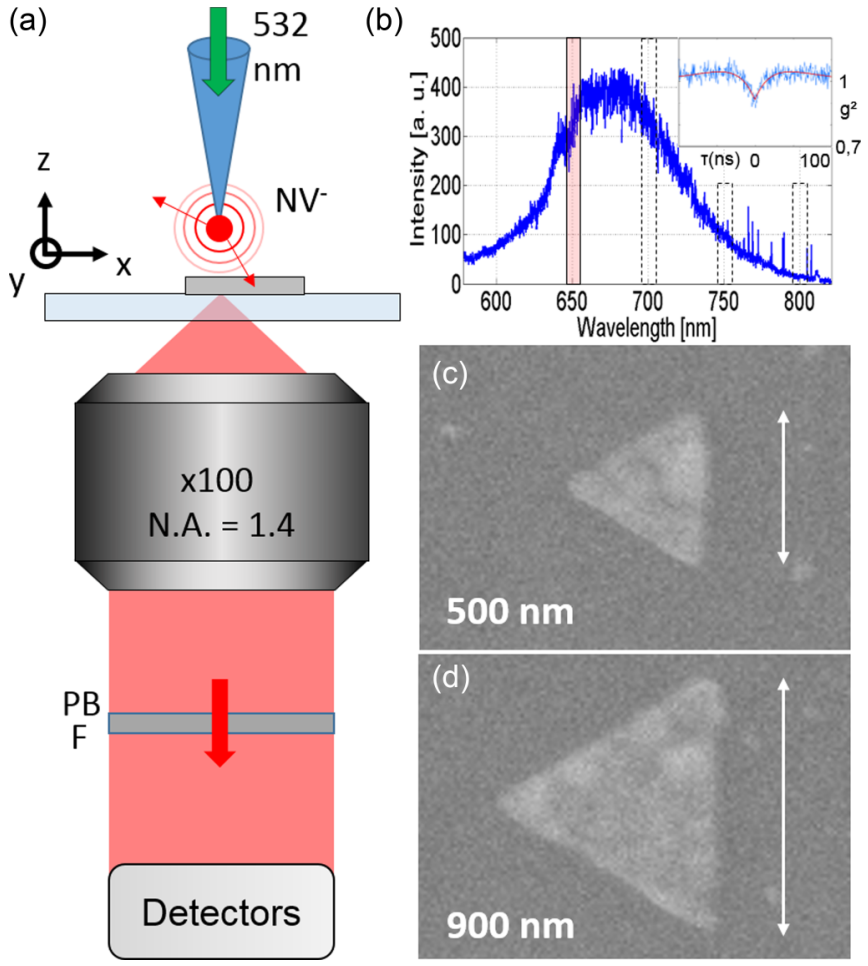

FIG. 1. (a) Schematic view of the experimental optical setup working in transmission. (b) Spectrum of the ten $\mathrm{NV}^{-}$colored centers hosted by the ND. The autocorrelation function of the emitting system is displayed in inset. The SEM images of a 500-nm and a 900-nm side long $2 \mathrm{D}$ triangular silver plasmonic cavities are respectively shown in (c) and (d).

energy released by an emitter at the tip apex and in a given selected frequency window $\Delta \omega$ centered around $\omega_{0}$ is given by (CGS units)

$$
\begin{aligned}
I\left(\mathbf{R}_{\mathrm{tip}}, \omega_{0}\right)= & \eta \int_{\omega_{0}-\frac{\Delta \omega}{2}}^{\omega_{0}+\frac{\Delta \omega}{2}} \hbar \omega P(\mathbf{v}, \omega) \Gamma\left(\mathbf{R}_{\mathrm{tip}}, \mathbf{v}, \omega\right) \\
& \times n_{2}\left(\mathbf{R}_{\mathrm{tip}}, \mathbf{v}, \omega\right) d \omega,
\end{aligned}
$$

where $\eta$ is a collection efficiency factor, $\mathbf{R}_{t i p}$ labels the position of the tip apex, and the unit vectors $\mathbf{v}$ have been introduced to define the orientations of the transition dipole moment $\mathbf{m}_{21}$, between the excited (2) and the ground state (1), with respect to an absolute surface frame. In addition, the three factors $P(\mathbf{v}, \omega), n_{2}\left(\mathbf{R}_{t i p}, \mathbf{v}, \omega\right)$, and $\Gamma\left(\mathbf{R}_{t i p}, \mathbf{v}, \omega\right)$, respectively, define an envelop function that accounts for the emitter frequencydependent spectrum, the population rate and the total decay rate of the excited state. These three quantities depend on the orientation and location of $\mathbf{m}_{21}$. The third one, $\Gamma(\mathbf{r}, \mathbf{v}, \omega)$ is given by

$$
\Gamma(\mathbf{r}, \mathbf{v}, \omega)=\Gamma_{0}+\frac{2 m_{21}^{2}}{\hbar} \operatorname{Im}\{\mathcal{S}(\mathbf{r}, \mathbf{r}, \omega)\}: \mathbf{v v} .
$$

with $\Gamma_{0}$ the decay rate in the vacuum considered constant over the $\Delta \omega$ window. The field-susceptibility $\mathcal{S}(\mathbf{r}, \mathbf{r}, \omega)$ that enters the total decay rate has been defined in Ref. [24]. This dyadic tensor describes the response of the whole particle-surface system.
In the case of an ND hosting several NV centers, the number of emitters and their random orientations have to be taken into account. According to experimental information [25], we assume that the laser power is sufficient to reach the saturated absorption regime for each $\mathrm{NV}^{-}$center.

We model the NV center as a three-level system with an metastable intermediate state (3). The population rate $n_{2}\left(\mathbf{r}_{j}, \mathbf{v}_{j}, \omega_{0}\right)=\gamma_{31} /\left(\gamma_{31}+\gamma_{23}\right)$ is obtained at saturation $[25,26]$. The latter expression shows that only nonradiative transition rates $\left(\gamma_{31}\right.$ and $\left.\gamma_{23}\right)$ associated with the metastable state (3) are involved. We therefore assume that the population rate of the excited state (2) is constant $n_{2}\left(\mathbf{r}_{j}, \mathbf{v}_{j}, \omega_{0}\right)=n_{2}$. Moreover, if we assume that the $p \mathrm{NV}$ centers in the ND bear randomly oriented transition dipoles and if we neglect any optical coupling between them, at a given energy, then Eq. (1) is simplified as

$$
I_{\text {tot }}\left(\mathbf{R}_{\text {tip }}, \omega_{0}\right) \cong \eta \Delta \omega n_{2} p \hbar \omega_{0} \bar{\Gamma}\left(\mathbf{R}_{\text {tip }}, \omega_{0}\right),
$$

where $\bar{\Gamma}\left(\mathbf{R}_{\text {tip }}, \omega_{0}\right)$ represents the total average decay rate per $\mathrm{NV}$ center that can be related to the ph-LDOS $\rho\left(\mathbf{R}_{\text {tip }}, \omega_{0}\right)$ experienced at the tip location $\mathbf{R}_{\mathrm{tip}}$ :

$$
\bar{\Gamma}\left(\mathbf{R}_{\text {tip }}, \omega_{0}\right)=\frac{4 \pi^{2} \omega_{0} m_{21}^{2}}{3 \hbar} \rho\left(\mathbf{R}_{\text {tip }}, \omega_{0}\right) .
$$

This whole emission phenomenon is associated either with an increase or a decrease of the local density of available photonic states generated near the metal structure. However, in the vicinity of dissipative materials, a second mechanism will modify the intensity $I_{R}\left(\mathbf{R}_{\text {tip }}\right)$ actually expected in the far-field zone, and Eq. (3) should be modified to include absorption mechanism by the metal [13]. This can be achieved by subtracting from $\bar{\Gamma}\left(\mathbf{R}_{O}, \omega_{0}\right)$ the average dissipated power $\bar{Q}\left(\mathbf{R}_{G}, \omega_{0}\right)$ in the metal per active NV center [27], leading to the expression for the experimental observable:

$$
I_{R}\left(\mathbf{R}_{\mathrm{tip}}\right)=\eta \Delta \omega n_{2} p \hbar \omega_{0}\left(\bar{\Gamma}\left(\mathbf{R}_{\mathrm{tip}}, \omega_{0}\right)-\bar{Q}\left(\mathbf{R}_{\mathrm{tip}}, \omega_{0}\right)\right) .
$$

The recent trend in molecular plasmonics has been to use single two-level systems which allow for a partial excitation/collection of the signal due to the arbitrary orientation of the associated pointlike dipole. It therefore limits, the relevant information because of the projection along the fixed dipole axis (for ph-LDOS mapping, for instance) [22,23]. In this work, the tip bears ten NV centers that comprise a collection of projections of the randomly oriented dipoles and turn the tip into a scalar probe. The temporal statistics of emitted photons of the $g^{(2)}$ autocorrelation function at zero delay reaches 0.9 [inset of Fig. 1(b)], which demonstrates that the quantum signature of the NV-based tip is preserved. This antibunching regime cannot be obtain with more classical active probes based on fluorescent beads because of the large number of emitters involved in the emission process [13].

While the discernible photons emitted by an NV center have been considered as a drawback for quantum experiments, it is actually a powerful tool for hyperspectral imaging in the near field [28] with the possible parallel acquisition of wavelength ranging from 640 to $800 \mathrm{~nm}$.

In order to compare the theoretical model with experimental data, we monitored the evolution of the fluorescence signal from ten $\mathrm{NV}^{-}$centers hosted by an $80-\mathrm{nm} \mathrm{ND}$ attached at 

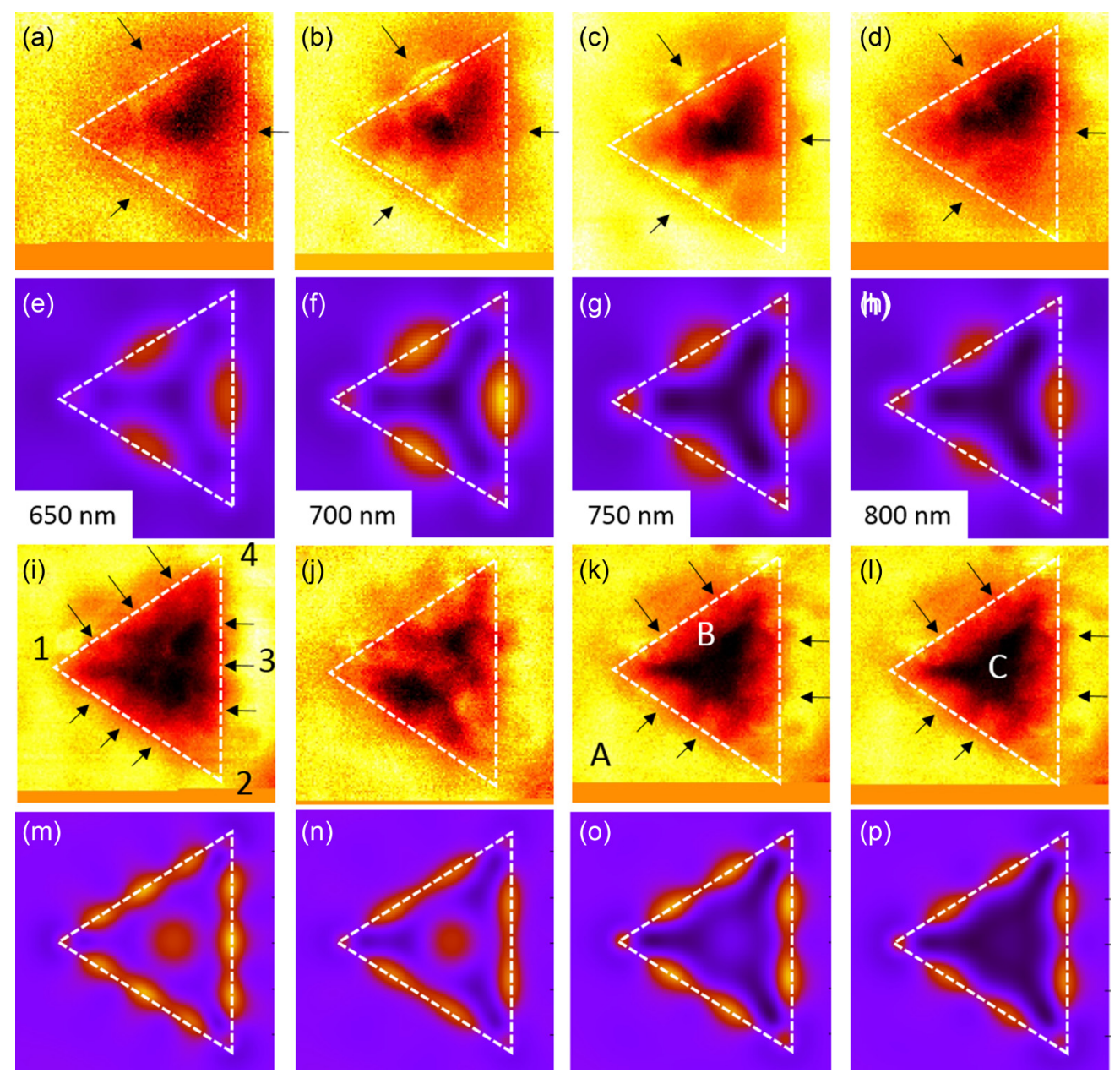

FIG. 2. (a)-(d) Fluorescence images recorded on top of the 500-nm-long prismatic cavity for different collection 10-nm-wide bandpass filters centered at 650 (a), 700 (b), 750 (c), and $800 \mathrm{~nm}$ (d). (e)-(h) Maps of the normalized radiative decay rate computed for scalar emitters positioned $40 \mathrm{~nm}$ above the structure and emitting at 650 (e), 700 (f), 750 (g), and $800 \mathrm{~nm}$ (h). (i)-(l) Fluorescence images recorded in similar conditions than (a)-(d) on top of the 900-nm-long cavity. Normalized radiative decay rate maps of (m)-(p), similar to (e)-(h) ones, for the 900-nm-long prismatic cavity.

the apex of a dielectric optical tip when driven into the near field of 2D multimodal mesoscopic plasmonic cavities made of silver [Fig. 1(a)]. The tip has been raster scanned above the structures at a constant height thanks to a shear-force based feedback [26]. It has recently been demonstrated that prismatic colloidal platelets sustain high-order delocalized SP modes in the visible that are essentially confined along the edges. The order of these modes can be tuned by changing the boundary conditions of the cavity (geometry or size) [29-31] resulting in a tailoring of the ph-LDOS in the direct vicinity of the structure too.

Aiming at probing their SP mode signatures, two equilateral triangles with lateral dimensions of 500 and $900 \mathrm{~nm}$ have been milled by focused ion beam (FIB) in a 30-nm-thick evaporated silver film [Figs. 1(c) and 1(d)]. The near-field fluorescence signals recorded with the ten NV centers based tip excited at $532 \mathrm{~nm}$ and raster scanned $40 \mathrm{~nm}$ above the structures are displayed in Fig. 2 for both cavity sizes. Four 10-nm bandpass filters centered at 650, 700, 750 and 800 have been selected to collect sequentially the signal in transmission through a high numerical aperture objective $(\mathrm{NA}=1.4)$. Images acquired on top of the 500-nm prism present contrasted features. First, the tip emits a constant fluorescence signal at a given energy when positioned far away from the structure, as expected for photostable NV centers. In contrast, the emission of the NV assembly is modified when approaching the cavity edges. In particular, for the four selected wavelengths, it translates into a nonvanishing signal above the structures all along the prism perimeter and more particularly by the emergence of bright lobes located above each edge middle [indicated by a black arrow in Figs. 2(a)-2(d)].

These effects are well reproduced when the computed normalized radiative contribution $\bar{\Gamma}_{R} / \Gamma_{0}\left(\propto I_{R} / I_{0}\right)$ of the decay rate for a scalar emitter [26] is compared to the experimental data at the corresponding wavelength shown in Figs. 2(e)-2(h) ( $I_{0}$ the intensity of the signal in the absence of the structure). The simulated maps exhibit the same lobes along the edges and moderate spots at each apex where the radiative part of the emission rate is accelerated. The intensity evolution of these lobes as a function of wavelength is identical in the experimental and simulated maps too with a maximal enhancement of the emission rate at 700 and $750 \mathrm{~nm}$. It is worth noting that the difference in contrast between experimental and simulated maps originates mainly from optical noise generated 
by dopants in the optical fiber and by the limited collection efficiency of the immersion objective.

The maps recorded above the 900-nm prism exhibit even richer patterns. The signal shows a higher number of maxima along the perimeter of the cavity that are indicated by black arrows. Interestingly, a small amount of fluorescence is also recorded at the cavity center at $\lambda=650 \mathrm{~nm}$ unlike above the small structure. The effect is even more pronounced when collecting at $700 \mathrm{~nm}$. More importantly, the hyperspectral analysis of the $\mathrm{NV}$ fluorescence reveals a modal jump at $\lambda=700 \mathrm{~nm}$ that translates into a variation of the number of identified antinodes along the edges. From three lobes recorded at $650 \mathrm{~nm}$, the system undergoes a modal transition that leads to the observation of only two lobes along each edges at $\lambda=750$ and $800 \mathrm{~nm}$. This behavior is exactly reproduced by our model that displays the same modal transition as the experiments. Notwithstanding the experimental resolution is limited by the finite size of the hosting ND $(80 \mathrm{~nm})$, the tip-sample distance $(40 \mathrm{~nm})$, the shear oscillation of the tip and the roughness of the silver structures and chromatic aberrations while the simulations correspond to a punctual emitter probing a perfectly flat structure, the modification of the radiative decay rate maps and the underlying ph-LDOS directly derive from the transition between an $m=5$ and an $m=6$ high-order SP mode in the cavity at these energies. Once immersed in the (a)

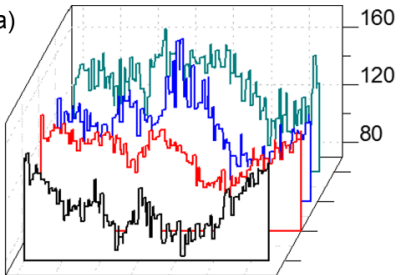

(c)

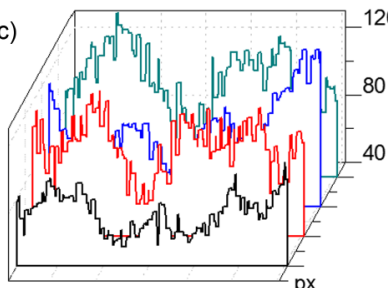

(e)

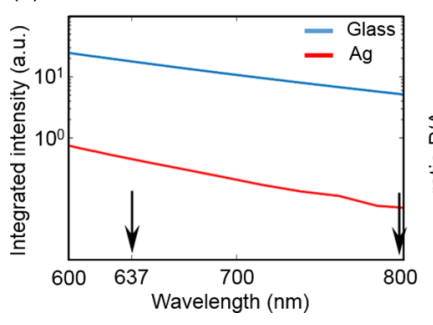

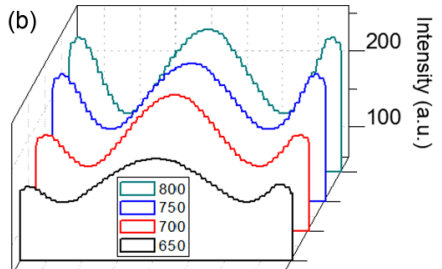

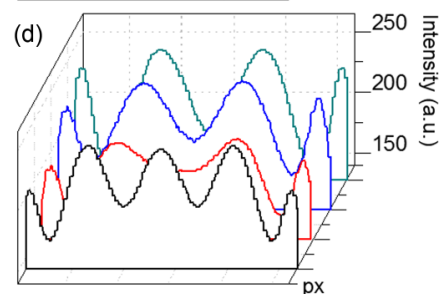

(f)

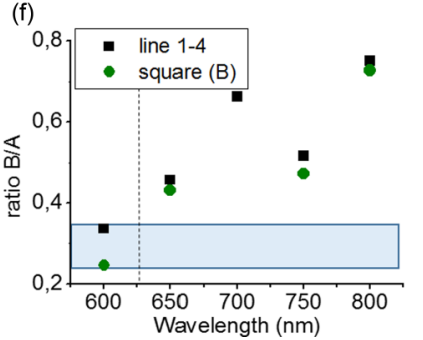

FIG. 3. (a) Experimental crosscuts done in the fluorescence intensity maps of the 500-nm prism in Fig. 2 and realized along the 1-4 axis [defined in Fig. 2(i)] at $\lambda=650$ (black), 700 (red), 750 (blue), and $800 \mathrm{~nm}$ (cyan). (b) Corresponding crosscuts extracted from simulated maps in Fig. 2. (c) and (d) are similar to (a) and (b) for the 900-nm long prismatic cavity. (e) Simulated integrated intensity collected in transmission through glass or $30 \mathrm{~nm}$ of silver originating from a scalar emitter $40 \mathrm{~nm}$ above the surface. (f) Ratio of the fluorescence intensities measured in A and B [defined in Fig. 2(k)], or along the 1-4 axis, as a function of the collection wavelength. structured near field, the dipole assembly experiences a strong modification of its photodynamics that follows the SP mode topology. Figure 3 provides complementary information from crosscuts in the experimental images [1-4 axis in Fig. 2(i)] that confirms the previous striking observations (a direct side-by-side experimental-simulation comparison is given in Ref. [26]). A single peak is observed in both Figs. 3(a) and 3 (b) for the 500-nm cavity while a transition from two to three maxima is established in Figs. 3(c) and 3(d) for the largest prism as the collection wavelength decreases.

In order to fully discard possible convolution effects and experimental artifacts near the prism edges, we compare the relative evolution of the fluorescence signal recorded above glass and above the cavity in the direct edge vicinity as a function of the wavelength [Figs. 3(e) and 3(f)]. The integrated light intensity emitted by a scalar emitter located $40 \mathrm{~nm}$ above a glass surface or a 30 -nm-thick silver film on glass is presented in Fig. 3(e) [32]. The curves show that the transmission through glass is obviously more efficient than on top of silver. The intensity computed downstream of the $\mathrm{Ag}$ film is quasi constant with a negligible wavelength dependence. It stems from the low dissipation in $\mathrm{Ag}$ as shown by the imaginary part of the dielectric function at these wavelengths. The transmission through silver being almost constant across the $\mathrm{NV}$ emission, the evolution of the ratio between $I_{R}$ measured in areas A and B [defined in Fig. 2(k)] as a function of the collection wavelength would be constant if our observations were due to imaging artifacts. As a comparison, we have measured such ratio by using the optical noise from the tip at $600 \mathrm{~nm}$ (outside of the $\mathrm{NV}$ emission range). The results in Fig. 3(f) show that this B/A ratio, measured in two different ways, is not constant as $\lambda$ increases. It can therefore only be explained by a local modulation of the wavelength dependent ph-LDOS near the metallic structure.

The good agreement observed between measured fluorescence intensity maps $I_{R}$ obtained by HQ-NSOM and $\bar{\Gamma}_{R}$ maps gives access to a radiative ph-LDOS as introduced earlier
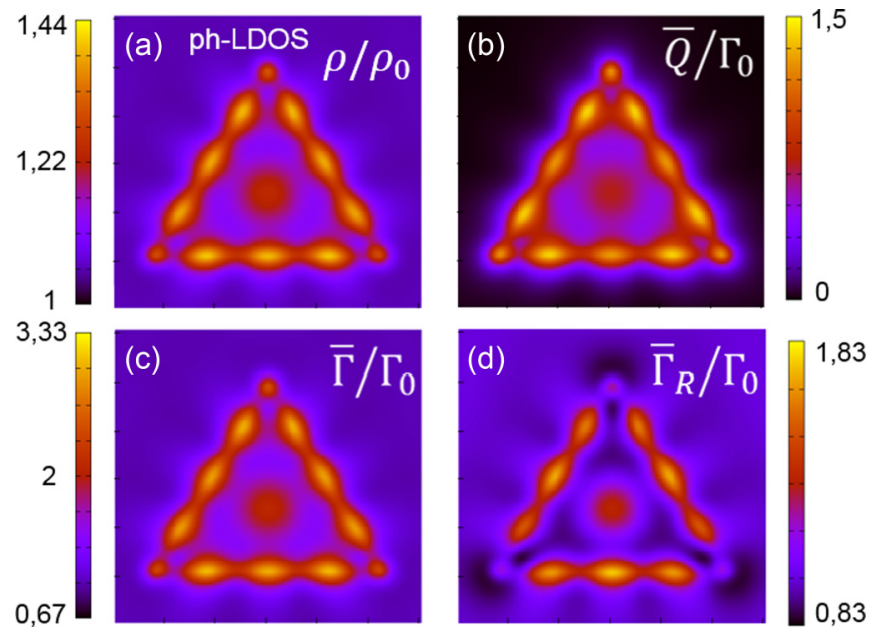

FIG. 4. (a) Simulated map of the total electromagnetic local density of states above the 900-nm-long cavity. (b) Normalized nonradiative decay rate map. (c) Normalized total decay rate map. (d) Normalized radiative decay rate map. The maps are computed at $\lambda=650 \mathrm{~nm}$. 
$[33,34]$. This formalism can thus be used to quantitatively assess the total ph-LDOS and the dissipative contribution $Q$ for any system investigated as shown in Figs. 4(a) and 4(b). Interestingly, as $Q$ depends on the square modulus of the electric field $|E|^{2}$ associated to the SP mode in the metal [27], it displays the same spatial dependence as the total decay rate and the ph-LDOS. This nonradiative enhancement has an amplitude comparable to the radiative one, meaning that the total decay rate is split into two equivalent contributions with half of the radiated energy lost in dissipative channels offered by the presence of the metallic nanostructure. The spatial matching between the two competing deexcitation pathways establishes the direct proportionality between HQ-NSOM signal $I_{R}$ and $\bar{\Gamma}_{R}$ which is in turn a better contrasted image of the total ph-LDOS once losses are taken into account.

To conclude, we have demonstrated that using a single ND hosting ten $\mathrm{NV}^{-}$centers attached at the apex of a sharp optical tip allows for hyperspectral photonic LDOS mapping. This tip acts as a scalar probe in a wide range of wavelength while preserving the quantum features of the emission statistics. HQ-NSOM imaging revealed the complex pattern of 2D multimodal plasmonic resonators with a resolution in energy sufficient to probe a modal jump in a submicrometer silver cavity. The comparison with a model built on the Green dyadic formalism confirmed the experimental observations and made possible the extrapolation of the radiative and nonradiative channels of the total decay rate, bridging experimental maps and ph-LDOS. This technique paves the way to a new kind of near-field experiments where ph-LDOS investigation and control as well as local quantum properties in the near field will have to be addressed.

This work was supported by the Agence Nationale de la Recherche (ANR) (Grant ANR-13-BS10-0007-PlaCoRe), and the massively parallel computing center CALMIP in Toulouse.
[1] M. S. Tame, K. R. McEnery, S. K. Özdemir, J. Lee, S. A. Maier, and M. S. Kim, Nat. Phys. 9, 329 (2013).

[2] F. D. Stefani, K. Vasilev, N. Bocchio, N. Stoyanova, and M. Kreiter, Phys. Rev. Lett. 94, 023005 (2005).

[3] S. Kuhn, U. Hakanson, L. Rogobete, and V. Sandoghdar, Phys. Rev. Lett. 97, 017402 (2006).

[4] G. P. Wiederrecht, J. E. Hall, and A. Bouhelier, Phys. Rev. Lett. 98, 083001 (2007).

[5] P. Anger, P. Bharadwaj, and L. Novotny, Phys. Rev. Lett. 96, 113002 (2006).

[6] H. Rigneault, J. Capoulade, J. Dintinger, J. Wenger, N. Bonod, E. Popov, T. W. Ebbesen, and P. F. Lenne, Phys. Rev. Lett. 95, 117401 (2005).

[7] A. V. Akimov, A. Mukherjee, C. L. Yu, D. E. Chang, A. S. Zibrov, P. R. Hemmer, H. Park, and M. D. Lukin, Nature (London) 450, 402 (2007).

[8] S. Schietinger, M. Barth, T. Aichele, and O. Benson, Nano Lett. 9, 1694 (2009).

[9] R. Kolesov, B. Grotz, G. Balasubramanian, R. J. Stöhr, A. A. L. Nicolet, P. R. Hemmer, F. Jelezko, and J. Wrachtrup, Nat. Phys. 5, 470 (2009).

[10] A. Huck, S. Kumar, A. Shakoor, and U. L. Andersen, Phys. Rev. Lett. 106, 096801 (2011).

[11] J. Michaelis, C. Hettich, J. Mlynek, and V. Sandoghdar, Nature (London) 405, 325 (2000).

[12] M. Frimmer, Y. Chen, and A. F. Koenderink, Phys. Rev. Lett. 107, 123602 (2011)

[13] D. Cao, A. Caze, M. Calabrese, R. Pierrat, N. Bardou, S. Collin, R. Carminati, V. Krachmalnicoff, and Y. De Wilde, ACS Photon. 2, 189 (2015).

[14] L. Aigouy, A. Caze, P. Gredin, M. Mortier, and R. Carminati, Phys. Rev. Lett. 113, 076101 (2014).

[15] A. Cuche, O. Mollet, A. Drezet, Y. Sonnefraud, O. Faklaris, F. Treussard, J.-F. Roch, and S. Huant, Opt. Express 17, 19969 (2009).

[16] A. Drezet, A. Cuche, and S. Huant, Opt. Commun. 284, 1444 (2011).

[17] G. Balasubramanian et al., Nature (London) 455, 648 (2008).
[18] A. Cuche, O. Mollet, A. Drezet, and S. Huant, Nano Lett. 10, 4566 (2010).

[19] M. Berthel, A. Drezet, and S. Huant, Opt. Lett. 41, 37 (2016).

[20] O. Mollet, S. Huant, G. Dantelle, T. Gacoin, A. Drezet, Phys. Rev. B 86, 045401 (2012).

[21] G. Colas des Francs, Ch. Girard, and A. Dereux, J. Chem. Phys. 117, 4659 (2002).

[22] R. Beams, D. Smith, T. W. Johnson, S.-H. Oh, L. Novotny, and A. N. Vamivakas, Nano Lett. 13, 3807 (2013).

[23] A. W. Schell, P. Engel, J. F. M. Werra, C. Wolff, K. Busch, and O. Benson, Nano Lett. 14, 2623 (2014).

[24] C. Girard, E. Dujardin, G. Baffou, and R. Quidant, New J. Phys. 10, 105016 (2008).

[25] M. Berthel, O. Mollet, G. Dantelle, T. Gacoin, S. Huant, and A. Drezet, Phys. Rev. B 91, 035308 (2015).

[26] See Supplemental Material at http://link.aps.org/supplemental/ 10.1103/PhysRevB.95.121402 for characterization of the tip, details on simulations, description of the 3-level system and for a side-by-side comparison of simulations and experiments along the edges and at the center of the cavities.

[27] G. Baffou, C. Girard, E. Dujardin, G. Colas des Francs, and O. J. F. Martin, Phys. Rev. B 77, 121101(R) (2008).

[28] J.-S. G. Bouillard, W. Dickson, G. A. Wurtz, and A. V. Zayats, ChemPhysChem 15, 619 (2014).

[29] L. Gu, W. Sigle, C. T. Koch, B. Ögüt, P. A. van Aken, N. Talebi, R. Vogelgesang, J. Mu, X. Wen, and J. Mao, Phys. Rev. B 83, 195433 (2011).

[30] S. Viarbitskaya, A. Teulle, A. Cuche, J. Sharma, C. Girard, E. Dujardin, and A. Arbouet, Appl. Phys. Lett. 103, 131112 (2013).

[31] S. Viarbitskaya, A. Teulle, R. Marty, J. Sharma, C. Girard, A. Arbouet, and E. Dujardin, Nat. Mater. 12, 426 (2013).

[32] A. Drezet and C. Genet, Phys. Rev. Lett. 110, 213901 (2013).

[33] C. Chicanne, T. David, R. Quidant, J. C. Weeber, Y. Lacroute, E. Bourillot, A. Dereux, G. Colas des Francs, and C. Girard, Phys. Rev. Lett. 88, 097402 (2002).

[34] G. Colas des Francs, C. Girard, M. Juan, and A. Dereux, J. Chem. Phys. 123, 174709 (2005). 